\title{
SOME RECENT DEVELOPMENTS IN THE AUDION RECEIVER ${ }^{1}$
}

$\mathrm{BY}$

Edwin H. Armstrong

THE AUDION AS DETECTOR AND AMPLIFIER

The fundamental operating characteristic of the audion is the relation between the wing current and the potential of the grid with respect to the filament-say the negative terminal of the filament. Such a characteristic is shown in Figure 1, and

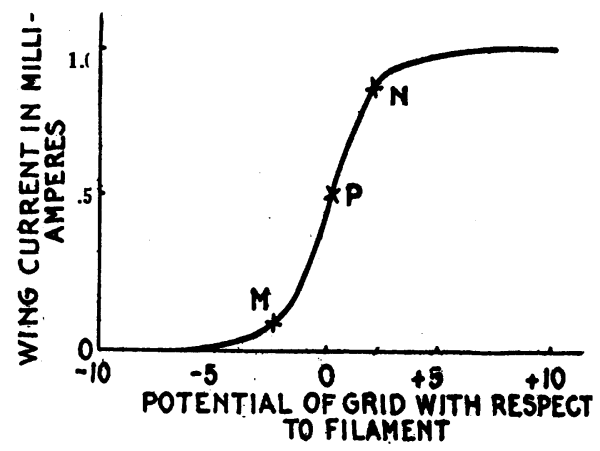

Figure 1

from it we see that a positive charge placed on the grid produces an increase in the wing current, and that a negative charge placed on the grid produces a decrease in the wing current. When the audion is used as an amplifier, and an alternating e. m. f. is impressed between the grid and the filament, the continuous current of the wing circuit will be varied in accordance

${ }^{1}$ Delivered before The Institute of Radio Engineers, New York, March 3, 1915, and before the Boston Section, April 29, 1915.

(The introductory material of this paper was originally submitted as a discussion by letter on Haraden Pratt's paper, "Long Range Reception with Combined Crystal Rectifier and Audion Amplifier." The first six figures have been kindly lent by the "Electrical World"; the remaining figures and text are herewith published for the first time.) 
with the characteristic of Figure 1, producing on the continuous current a superimposed a. c. wave in phase with and of the same frequency as the impressed e.m.f. Diagrammatically this action is shown in Figure 2.

The action of the audion as a detector of radio frequency oscillations is very different from its action as a simple amplifier. Some form of connection must be used, such that the effect of a

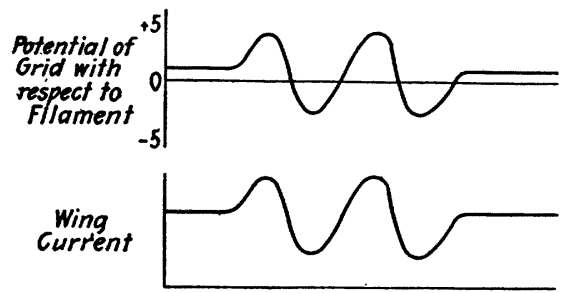

Figure 2

group of radio frequency oscillations in the grid circuit of the audion is translated into a single audio frequency variation of the current in the telephones. The usual method is to make use of the valve action between the hot and cold electrodes at low pressures, and the connection used to do this is shown in Figure 3. In this method of connection there are two distinct

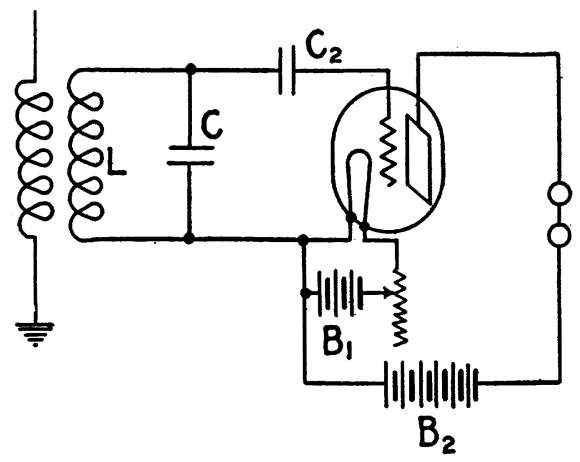

Figure 3

actions; one rectifying and the other amplifying. The closed oscillation circuit: LC, filament, grid, and condenser $\mathrm{C}_{2}$, behaves exactly as a Fleming valve receiver, the incoming oscillations being rectified between the grid and filament and the rectified current being used to charge the condenser $\mathrm{C}_{2}$ (the side connected to the grid being of course negative). The negatively 
charged grid then exerts a relay action on the wing current, decreasing it; the wing current returning to its normal value as the charge in the grid condenser leaks off by way of the grid and the grid resumes its normal potential. If the audion is properly constructed, the relay action results in an amplification of the energy available for use in the telephones over that which would be available in a simple rectifier. Figure 4 indicates the features of the valve method of detection.

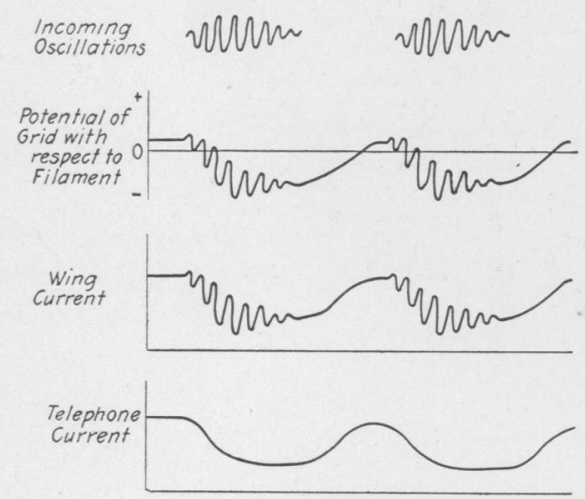

Figure 4

Working in conjunction with Professor Morecroft, I have recently secured oscillograms which confirm the explanations already advanced and these oscillograms and the means by which they were obtained are herewith shown in Figures 5, 6 and 7.

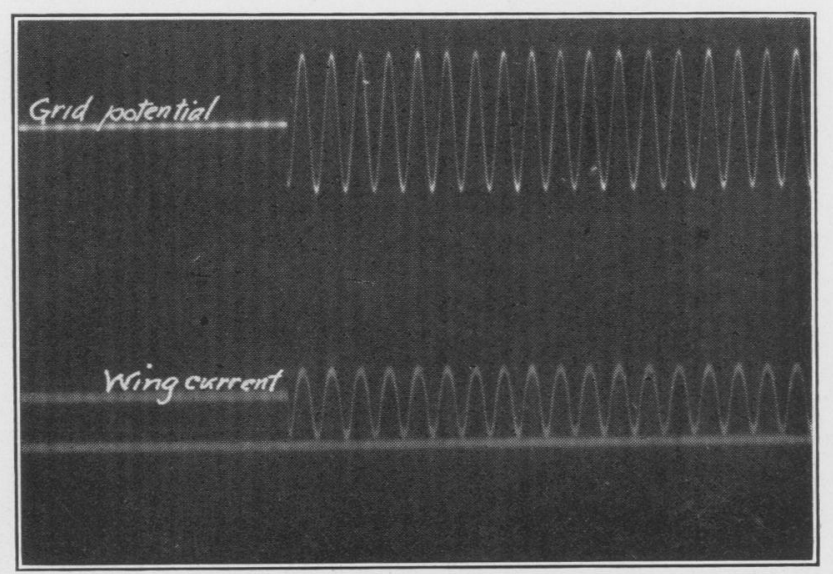

Figure 5 
It will be seen, therefore, that using the audion as a detector of radio frequency oscillations, it has been shown that in addition to operating as a rectifier it simultaneously acts as a

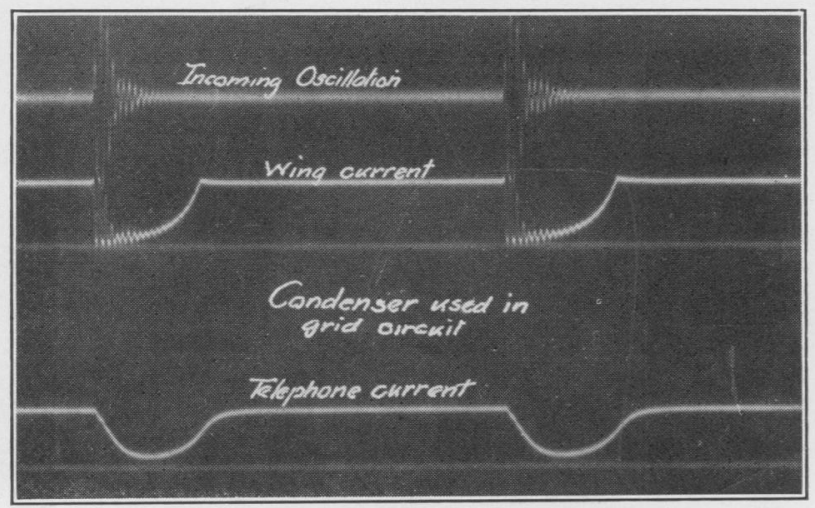

Figure 6

repeater of the radio frequencies; so that oscillations in the grid circuit set up oscillations of similar character in the wing circuit of the audion. In the ordinary detector system no use

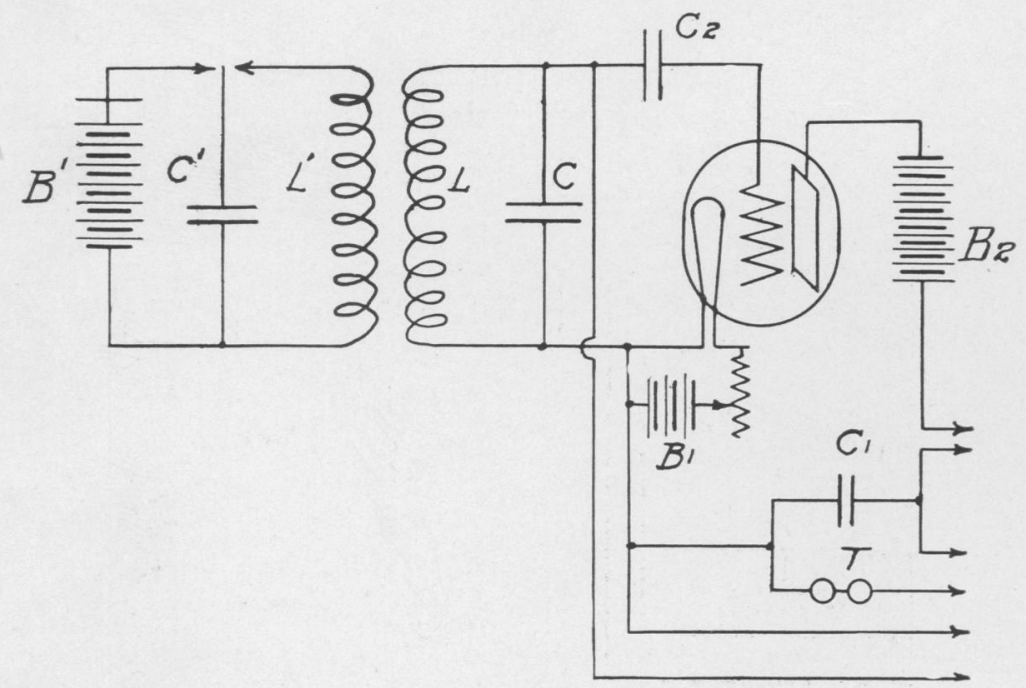

Figure 7

is made of the repeating action, and it is the purpose of the present paper to show that it may be turned to account to produce improvements in the reception of signals which com- 
pletely overshadow any of the particular advantages of the audion when used as a simple detector. The ordinary detector circuit is illustrated by Figure 3 and the phenomena present therein may be summed up diagrammatically by the curves of Figure 4. It will be seen from these that the radio frequency oscillations present in the wing circuit of Figure 3 with the ordinary audion are necessarily small and also that they are of no value in producing a response in the telephones; but by providing means for increasing their amplitude and means for utilizing them to reinforce the oscillations of the grid circuit, it becomes possible to produce some very remarkable results.

\section{REINFORCEMENT OF RADIO FREQUENCY OSCILLATIONS BY THE AUDLON}

There are two ways of reinforcing the oscillations of the grid circuit by means of those in the wing circuit. The simplest way perhaps is to couple the two circuits together in the manner shown in Figure 8. This is essentially the same as Figure 3, but

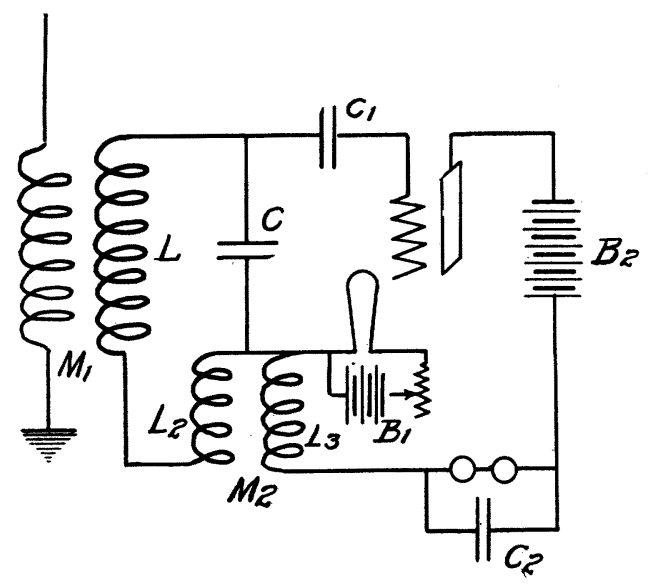

Figure 8

modified by the introduction of the inductively coupled coils $\mathrm{L}_{2}$ and $\mathrm{L}_{3}$ in the grid and wing circuits respectively and by the condenser $\mathrm{C}_{2}$ which forms a path of low impedance across the telephones for the radio frequencies. In such a system, incoming signals set up oscillations in the grid circuit which repeat into the wing circuit producing variations in the continuous current, the energy of which is supplied by the battery $B_{2}$. By means of the coupling $\mathbf{M}_{2}$, some of this energy of the wing oscillations is transferred back to the grid circuit, and the 
amplitude of the grid oscillations thereby increased. The amplified grid oscillations then react on the wing circuit by means of the grid to produce larger variations in the wing current, thus still further reinforcing the oscillations of the system. Simultaneously with this procedure the regular detecting action goes on; the condenser $\mathrm{C}_{1}$ is charged in the usual way, but accumulates a charge which is proportional, not to the original signal strength but to the final amplitude of the oscillations in the grid circuit. The result is an increased response in the telephone proportional to the energy amplification of the original oscillations in the grid circuit. It will be observed from the operating characteristic (the relation between grid potential and wing current), that the amplitude of the variation in the wing current is directly dependent on the variation of the grid potential. This indicates that the grid circuit should be made up of large inductance and small capacity to obtain the maximum voltage which it is possible to impress on the grid. For moderate wave lengths the tuning condenser $\mathrm{C}$ of the grid circuit may be omitted altogether and the capacity of the audion alone used to tune the circuit. For long wave lengths, the distributed capacity of the grid circuit inductance becomes so high with respect to the capacity of the audion that better results are obtained by the use of a tuning condenser to fix definitely the points of maximum potential difference across the grid and filament of the audion.

In the second method of reinforcing the oscillations of the grid circuit the wing circuit of the audion is tuned by means of an inductance introduced as shown by Figure 9. This differs

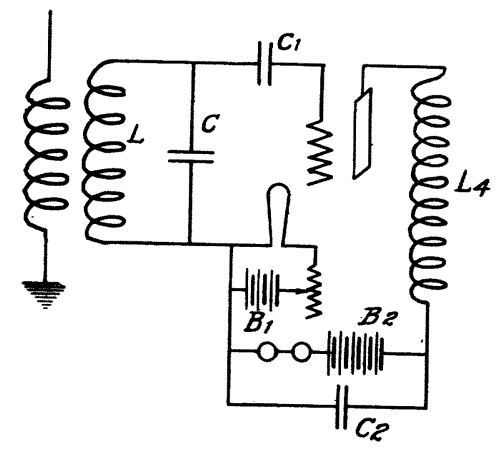

Figure 9

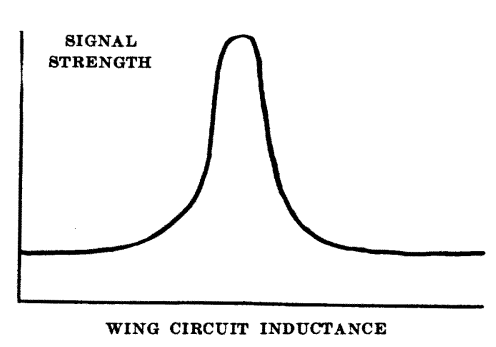

Figure 10

from the ordinary detector circuit of Figure 3 by the addition of the coil $\mathrm{L}_{4}$ and the condenser $\mathrm{C}_{2}$. The manner in which the 
grid oscillations are amplified may best be understood by the following analysis. With no oscillations in the system, the potential difference between filament and wing will be approximately the voltage of the battery $\mathrm{B}_{2}$, but when oscillations are set up in the grid circuit, causing radio frequency variations of the wing current, the potential of the wing with respect to the filament varies as the reactance voltage of the wing inductance alternately adds to and subtracts from the voltage of the battery. When a negative capacity charge is placed on the grid, the wing current will be reduced and the direction of the reactance voltage of the wing inductance will therefore be the same as the voltage of the battery $\mathrm{B}_{2}$. The reactance voltage will therefore add to the battery voltage and the difference of potential between wing and filament and also between wing and grid will be increased. Similarly when a positive charge is placed on the grid the wing current is increased and the reactance voltage of the wing inductance opposes the battery voltage, producing a decrease in the potential difference between grid and wing. Hence, supposing a negative capacity charge is placed on the grid, the tendency of the corresponding increase in the potential of the wing with respect to the grid will be to draw more electrons out on the grid, thereby increasing the charge in the condenser formed by the wing and grid, the energy for supplying this charge being drawn from the wing inductance as the wing current decreases. The increased negative charge on the grid tends to produce a still further decrease in the wing current and a further discharge of energy from the wing inductance into the grid circuit. On the other hand, when a positive charge is placed on the grid, the potential difference between grid and wing is reduced and some of the energy stored in the capacity formed by them is given back to the wing inductance. During this part of the cycle, electrons are being drawn into the grid from the surrounding space to charge the grid condenser in accordance with the well known valve action, and this, in effect, is a conduction current, so that a withdrawal of energy from the circuit takes place. In spite of this withdrawal of energy, however, a well defined resonance phenomena between the audion capacity and the wing inductance is to be expected and in the reception of signals such is found to be the case. When the wing inductance is properly adjusted at the resonance frequency, energy from the wing circuit is transferred freely to the grid circuit and oscillations build up therein and are rectified in the usual way. 
A curve showing the general relation between signal strength and value of wing inductance is shown in Figure 10, the circuits used being those of Figure 9. As the capacity of the audion is the main means of transferring energy from the wing to the grid circuit, best results are obtained when the condenser $\mathrm{C}$ is very small. On account of the very small capacity of the audion, the eff ectiveness of this method of tuning is more pronounced at the higher frequencies, but by the use of a shunt condenser across the inductance of the wing circuit very good amplification is secured on frequencies as low as 30,000 cycles $(10,000$ meters wave length). The best results, however, are obtained with some combination of coupling and wing circuit tuning, as il-

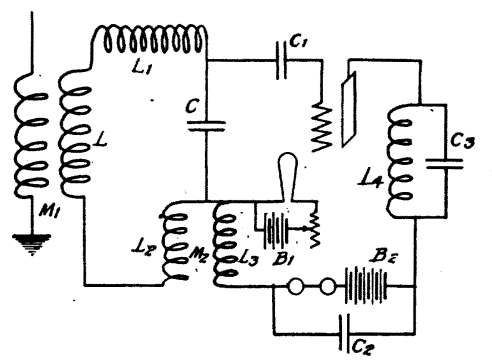

FIGURE 11

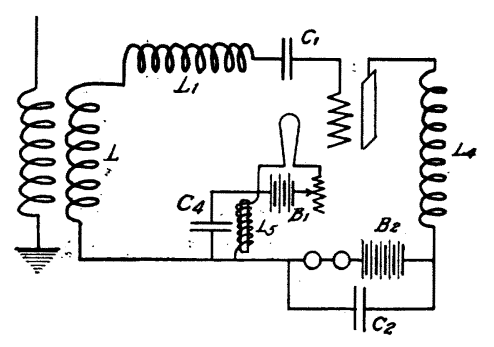

Figure 12

lustrated in Figure 11. Other methods of coupling may be employed between the grid and wing circuits, electrostatic and direct magnetic couplings being illustrated in Figures 12 and 13. The arrangement of Figure 13 operates in the same way as the system with the two coil coupling; but the electrostatic coupling

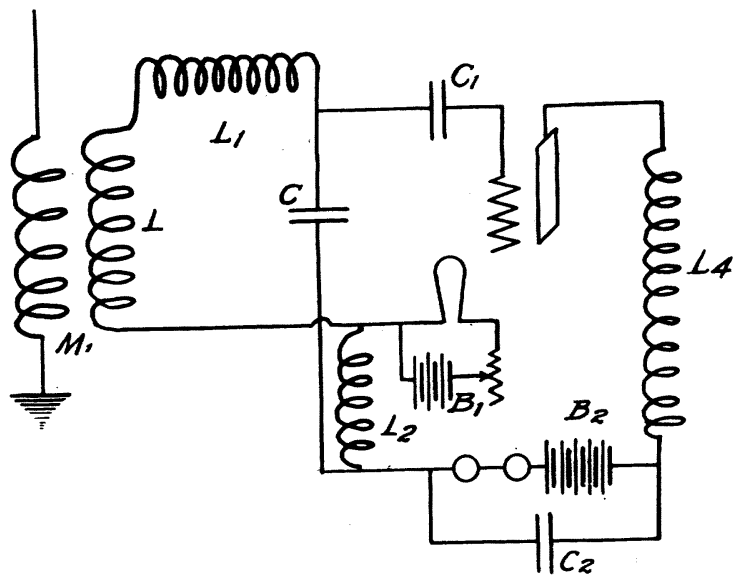

FIGURE 13 
of Figure 12 works in an odd way. It is necessary, in this connection, to complete the wing circuit for the continuous current of the battery and this is done by shunting the coupling condenser $\mathrm{C}_{4}$ by a coil of high inductance. The continuous current of the wing circuit flows thru this coil and $\mathrm{C}_{4}$ provides a path of low impedance around this coil for the radio frequency oscillations of both grid and wing circuits. When a positive charge is placed on the grid, an increase in the wing current results, the alternating component of the wing current charging the condenser $\mathrm{C}_{4}$ and the sum of the currents passing thru $\mathrm{C}_{4}$ and $\mathrm{L}_{4}$ equalling the current thru the audion. When a negative charge is placed on the grid the current thru the audion is reduced and the inductance $L_{5}$ discharged into the condenser shunted across it, charging it in the opposite way to that caused by the increase in the wing current. In both cases, $\mathrm{C}_{4}$ then diseharges thru the grid circuit reinforcing the oscillations therein.

\section{AUDIO FREQUENCY AMPLIFICATION}

It is possible to combine with any of these systems a system of audio frequency circuits which amplify the telephone current in exactly the same manner as the radio frequency oscillations are amplified, and such a system is shown in Figure 14. Here $\mathbf{M}_{2}$

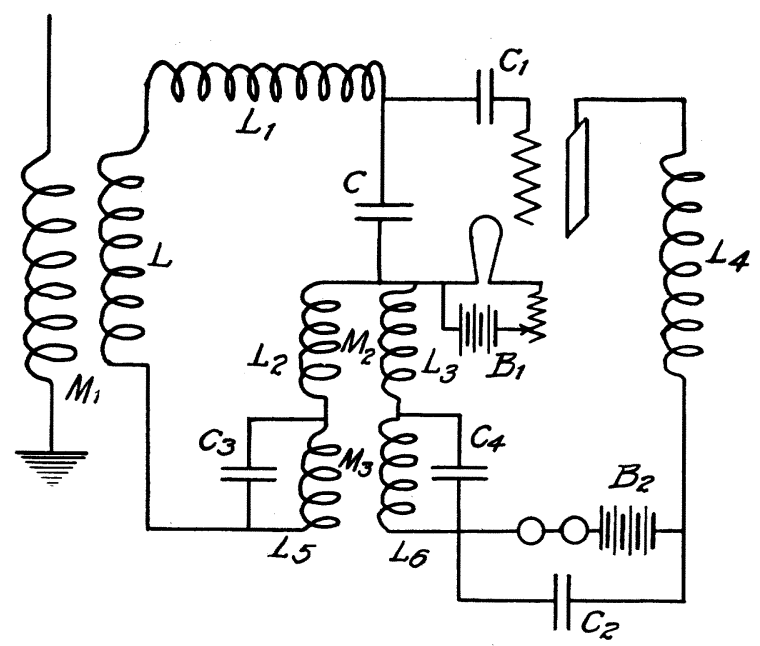

FIgURe 14

represents the coupling for the radio frequencies and the coils are of relatively small inductance. $\mathrm{M}_{3}$ is the coupling for the audio frequencies, and the transformer is made up of coils having an inductance of the order of a henry or more. The condensers $\mathrm{C}_{3}$ and 
$\mathrm{C}_{4}$ have the double purpose of tuning $\mathrm{M}_{3}$ to the audio frequency, and of by-passing the radio frequencies. The total amplification of weak signals by this combination is about 100 times, with the ordinary audion bulb. On stronger signals, the amplification becomes smaller as the limit of the audion's response is reached.

\section{THE AUDION AS A GENERATOR AND BEAT RECEIVER}

Any repeater, which is also an energy amplifier, may be used to produce continuous oscillations by transferring part of the energy in the circuit containing the battery back to the controlling circuit to keep the latter continuously excited. By providing a close enough coupling between the grid and wing circuits, sufficient energy is supplied to the grid circuit to keep it in continuous oscillation, and as a consequence thereof oscillations of similar frequency exist in all parts of the system. The frequency of these oscillations is approximately that of the closed grid circuit if the tuning condenser of that circuit is large with respect to the capacity of the audion. If this capacity is small, then the wing circuit will exert a greater influence on the frequency of the system, and it will not approach that of the grid circuit so closely. When such a system of circuits is in oscillation, it has been found possible not only to receive continu-

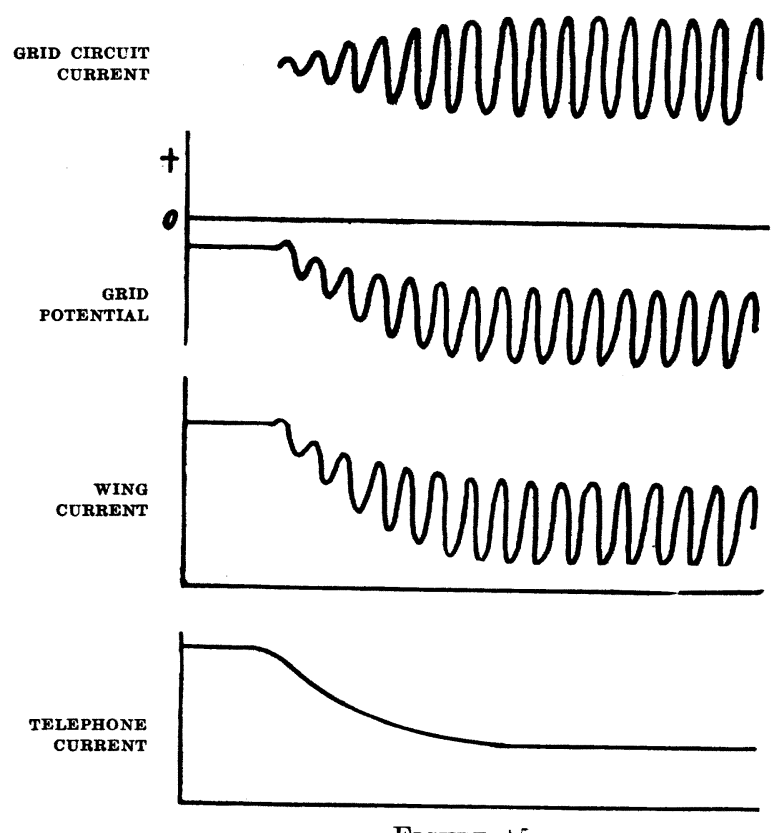

Figure 15 
ous waves by means of the beat method but also very greatly to amplify them as well.

The phenomena involved may best be understood by reference to Figures 15 and 16, which show the relation between wing
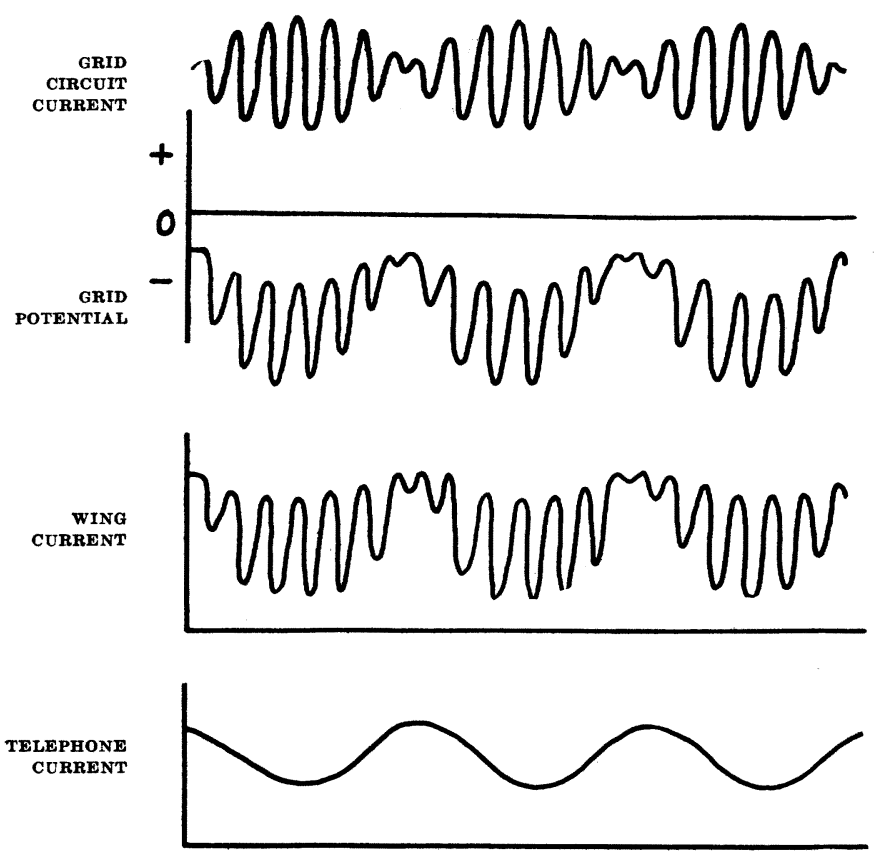

Figure 16

current and time at the beginning of oscillation. When the audion begins generating, the grid oscillations are continuously rectified to charge the grid condenser, and this charge continuously leaks off either by way of the grid or by means of a special high resistance placed in shunt with the condenser. As the negative charge builds up in the grid condenser, it decreases the average value of the continuous current component of the wing current and therefore limits the amplitude of the oscillations of the grid circuit until a point is finally reached where the rate at which electricity is supplied to the grid condenser is just equal to the rate at which it leaks off. Consider now the effect on the system of an incoming continuous wave having a frequency slightly different from the frequency of the local oscillations. The presence of the local oscillations will not in any way interfere with the amplifying powers of the system and the incoming oscillations will build up in exactly the same manner as for the 
non-oscillating state but to a greater degree because of the closer grid and wing coupling. Simultaneously with the amplifying of the incoming wave, beats are produced between the local and the signalling currents, the effect being alternately to increase and decrease the amplitude of the oscillations in the system. From Figure 15 it will be apparent that when this steady state is reached an increase in the amplitude of the grid oscillations by any means whatever will increase the negative charge in the grid condenser, producing a decrease in the average value of the wing current and hence a decrease in the telephone current. On the other hand, a decrease in the amplitude of the oscillations will allow some of the negative charge in the grid condenser to leak off and thereby permit an increase in the telephone current. Hence, when incoming and local oscillations add up, the negative charge in the grid condenser is increased and a decrease in the telephone current results. When the two frequencies are opposed, some of the charge in the grid condenser leaks off and an increase in the telephone current occurs. The result is the production in the telephones of an alternating current having a frequency equal to the difference in the frequencies of the local and incoming oscillations and having the very important property of being almost simple harmonic. Figure 16 illustrates the characteristics of this method of reception. The complete phenomena may be summed up as follows. Incoming oscillations are simultaneously amplified and combined in the system to produce beats with a local oscillation continuously maintained by the audion. The radio frequency beats are then rectified by the audion to charge the grid and the grid condenser, and this charge varies the electron current to produce an amplifying action on the current in the telephones.

When the grid condenser is omitted, the beat phenomenon is slightly modified, and the audio frequency variation of the telephone current is produced according to the asymmetric action outlined in a previous publication dealing with the operating features of the audion. The system is more sensitive with the grid condenser, but the same general result is obtained by either method of reception.

\section{PECULIAR FEATURES OF OSCILLATION}

Some very interesting features of operation accompany the production of oscillations in the system. Suppose the audion is not oscillating, and the grid and wing coupling is fairly weak. As this coupling is increased, the point at which oscillations 
begin is indicated by a faint click in the telephones accompanied by a slight change in the character of the static. The oscillations produced are usually so high in frequency and constant in amplitude that they are entirely inaudible. As the coupling is still further increased, a rough note is heard in the telephones the pitch decreasing with increase of coupling. This note is produced by the breaking up of the oscillations into groups, and it occurs whenever electricity is supplied to the grid condenser at a greater rate than that at which it can leak off. The result is that the grid is periodically charged to a negative potential sufficient to cut off entirely the wing current, causing a stoppage of the local oscillations until the grid charge leaks off and the wing current re-establishes itself. The frequency of this interruption depends largely on the capacity of the grid condenser, the resistance of its leakage path, and the amplitude of the local oscillations; and it may be varied from several hundred down to one or less per second. This effect is sometimes troublesome in the reception of signals, especially with high vacuum tubes. It may be eliminated, however, by increasing the leak of the grid condenser by means of a high resistance shunt. The best coupling for receiving continuous waves lies somewhere between the point at which oscillations start and the point at which interruption begins, and can only be determined by trial. In this region, trouble is sometimes experienced by the appearance of a smooth musical note in the telephones. This occurs under certain critical conditions of coupling with the antenna when the grid circuit oscillates with two degrees of freedom. Two slightly different frequencies are therefore set up, producing beats which are rectified by the audion in the usual way. This effect is quite critical, and when it causes

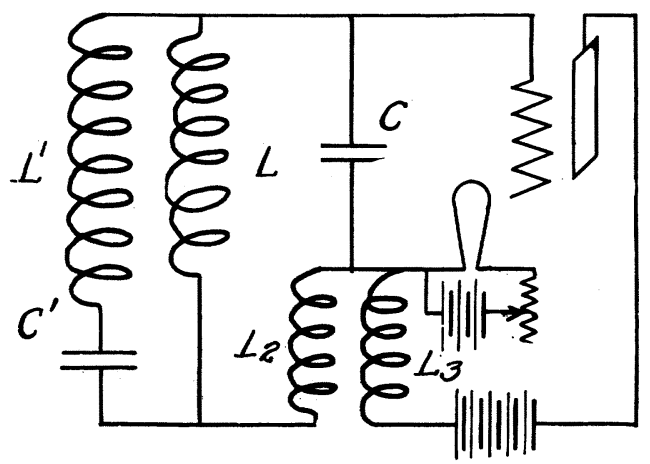

Figure 17 
interference with signals, a slight readjustment of the circuits will usually make it disappear. It may, however, be made perfectly steady and reproduced at will by the system shown in Figure 17, where two grid circuits of different periods are provided. Two frequencies are therefore generated one having the frequency of the circuit $\mathrm{LCL}_{2}$, and the other the frequency of the circuit $\mathrm{L}^{\prime} \mathrm{C}^{\prime} \mathrm{L}_{2} \mathrm{C}$. This arrangement may replace to advantage the ordinary buzzer for producing groups of oscillations. The foregoing explanations refer to the audion only when it is used as an electron relay. ${ }^{*}$ When there is an appreciable amount of gas, in the tube in the ionized state, disturbances of an entirely different character occur.

\section{AUDIO FREQUENCY TUNING}

One of the very important advantages of the receiver when used for continuous waves is that the alternating current produced in the telephones is almost a pure sine wave. Only when the audio frequency is simple harmonic can selectivity be obtained by tuning the telephone circuit. A distorted wave such as that produced by spark signals possesses many harmonics and as each may be picked out by the tuned telephone circuit there is little chance of separating two spark signals by audio frequency tuning. With continuous waves, however, the pure wave produced by the beat method of reception makes it possible to obtain selectivity by the audio frequency tuning, resonance

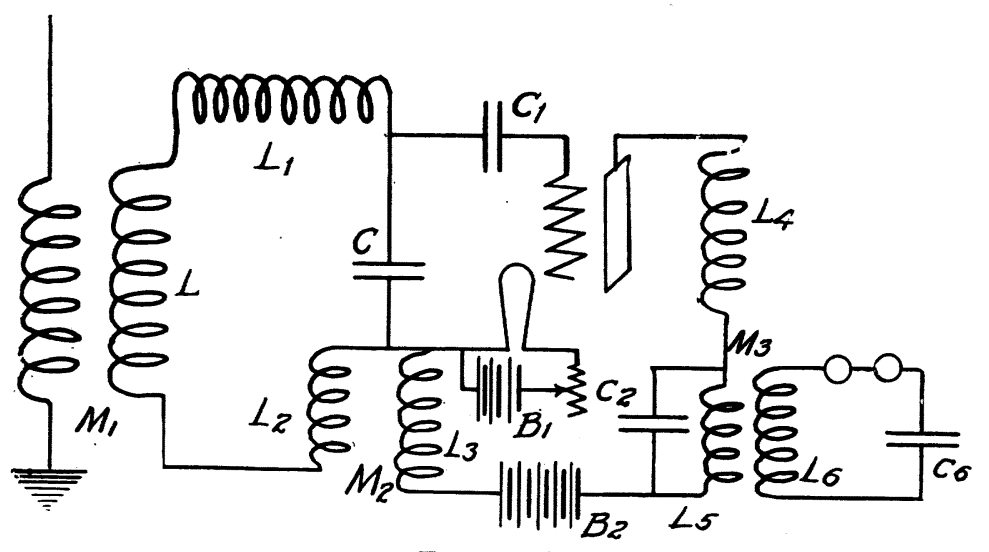

FigURe 18

* "Electrical World," December 12, 1914; and also discussion in "London Electrician," between Reisz and de Forest on the difference between electron and gas relays. (February 6, 1914, page 726; March 13, 1914, page 956; June 12 , 1914, page 402 ; July 3 , 1914, page 538 ; and July 31 , 1914, page 702 .EDITOR.) 
being fully as sharp as in the radio frequency circuits. Two methods of audio frequency tuning are shown in Figures 18 and 19. In Figure 18, the telephone is inductively connected to the wing circuit of the audion by means of a transformer the secondary of which includes besides the telephone a tuning condenser.

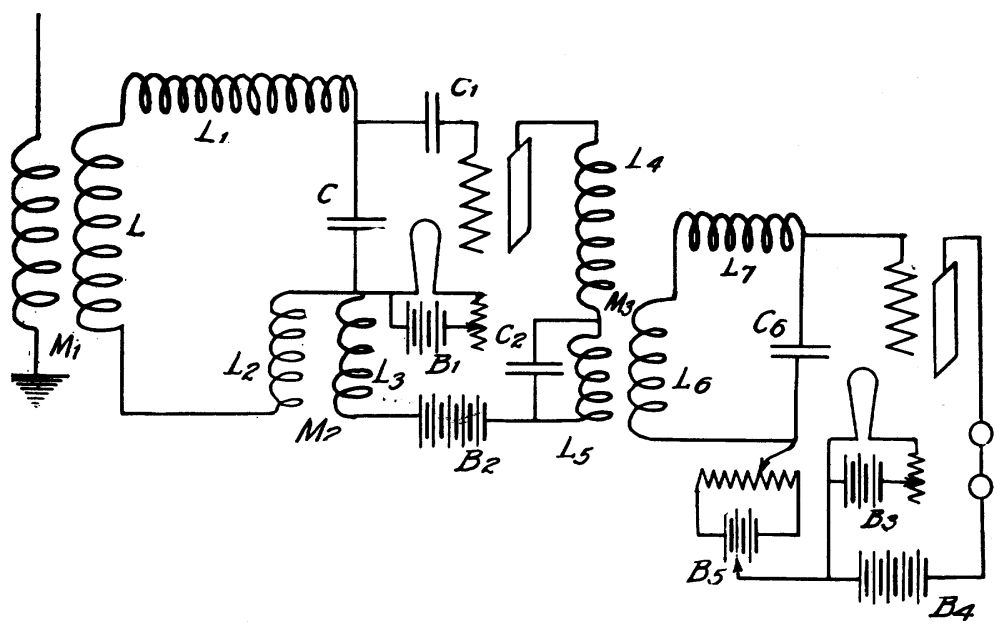

Figure 19

In this connection, the telephone, with a resistance of many thousand ohms, is placed directly in the tuned audio frequency circuit, and hence for good tuning the inductance of the coil $\mathrm{L}_{6}$ must be made extremely large to secure the necessary ratio of the reactance of $L_{5}$ to the resistance of the circuit. This disadvantage is overcome in the system of Figure 19 by removing the telephones from the audio frequency circuit, and using the latter to operate a second audion. The telephones may then be placed in the wing circuit of this audion without adding appreciably to the damping of the circuit. The tuning of the circuit $\mathrm{L}_{6} \mathrm{C}_{6}$ may therefore be made very sharp with reasonable values of inductance simply by keeping the resistance low. In this case considerable amplification is obtained by the use of resonance in the transformer $M_{3}$ to increase the voltage impressed on the grid of the second audion. The great advantage of this kind of tuning is shown by the following example. Suppose the incoming signal has a frequency of 50,000 cycles, and the local frequency is 49,000 cycles. The differential frequency is 1,000 , and the audio frequency circu t is tuned accordingly. An interfering wave 1 per cent. shorter than the signalling wave, or 49,500 cycles, will produce an audio frequency of 500 cycles per second, 
which will not appear at all in the wing circuit of the second audion unless it is many times stronger than the 1,000 cycle signal. This combination of radio and audio frequency tuning is too selective for use at the present time even when the sending station is equipped with an alternator, as the slight changes in frequency of the radiated wave produce changes in the beat frequency of the receiver which carry it out of range for the sharply tuned audio frequency circuit. A disadvantage of this method of tuning is that atmospheric disturbances produce a musical note due to shock excitation of the audio frequency system. Very loose coupling with the wing circuit of the first audion is a partial remedy for this. There are times, however, when interference is more troublesome than static and in such cases the method may be used to great advantages. If desired, both radio and audio frequency tuning can be carried out in the same audion as indicated in Figure 14. This combination is apt to be somewhat troublesome to operate as a cumulative amplification is obtained in the audio frequency as well as in the radio frequency system.

\section{CASCADE SYSTEMS}

Where a greater amplification than can be obtained with one audion is required, cascade working of the radio frequency systems may be resorted to by coupling together two or more audion systems, each connected as already described, in the manner indicated in Figure 19. The incoming oscillations in the first audion system are amplified in the usual manner and

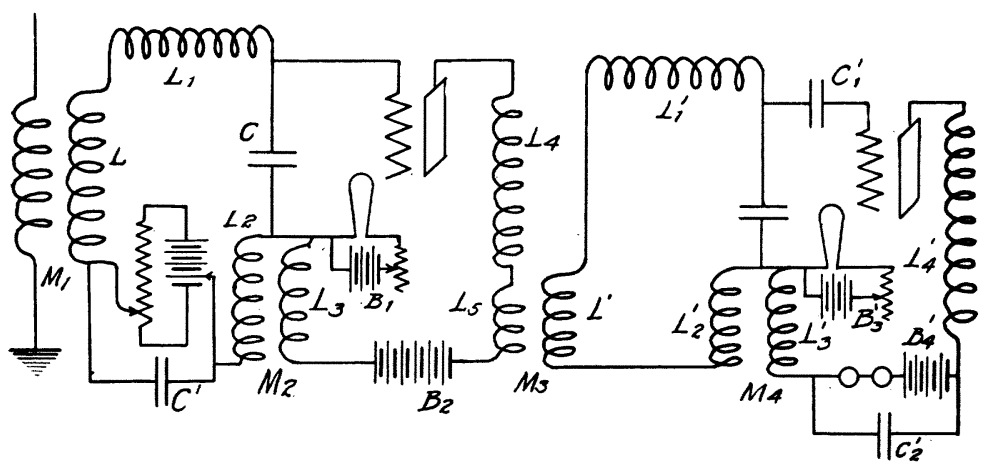

FIgURE 20

set up oscillations in the second system by means of the coupling $\mathrm{M}_{3}$ (See Figure 20). The oscillations initially set up in the second system are again amplified, and then rectified in the second audion to produce audible response in the telephones. 
For the reception of spark signals, considerable adjustment is required to get the best results without causing one or the other or both of the systems to generate oscillations. It will be found that after the first circuit is adjusted to the point of oscillation and the second is coupled with it, the strength of signal in the first system will be reduced owing to the withdrawal of energy from it by the second system. The signals may then be again brought up in strength by increasing the coupling between the grid and wing circuits of the first audion until the appearance of the local oscillations indicates that the limit of amplification has been reached. By careful adjustment about a thousand times amplification and very sharp tuning can be obtained with two steps.

For continuous wave reception, there are several methods of operating cascade systems. It is possible to have either system generate oscillations, the other system acting simply as an amplifier or both systems may be made to generate in synchronism. It will generally be found that when both systems produce oscillations, beats will be produced, so that a continuous note is heard in the telephones; but by adjusting the frequency of one of the systems the pitch of this note will be reduced as the two systems approach synchronism, until finally at one or two hundred beats per second the two systems pull into step in much the same way as two alternators. The ability of the two systems to keep in step depends mainly on the value of the coupling between them, and the closer this is the better the two hold together. There is still another way of working this combination, and that is asynchronously. In this case beats are continuously produced in the system so that a continuous note is heard in the telephone, but the circuits may be so adjusted that the note is not loud enough to be troublesome or it may be tuned out of the telephone in the manner previously described. Incoming oscillations are combined in the system to produce beats with the beats already present so that a rather curious note is heard. Very good amplification is secured by this method though naturally the system is troublesome to operate.

It may be noted here that whenever a signal is too weak to read with one audion system and cascade operation becomes necessary, it is always better practice to use the cascade circuits for the radio frequencies, even if the regenerative circuits are not employed with each individual audion system. The frequency of the oscillations set up in the circuits by static are, 
under normal conditions, the same as those of the incoming signal; and the static is therefore never amplified more than the signal. Usually it is amplified to a somewhat lesser extent, especially if regenerative circuits are employed. In the cascade systems used for audio frequencies, a different condition exists. It is ordinary practice to connect the different stages by means of transformers, and this leads to conditions which cause the system to produce greater amplifications of the higher frequencies. The rate of change of the wing current of the detecting audion produced by static corresponds to a very high frequency, and as such is invariably amplified to a greater extent than the signal.

There is a second method of receiving continuous oscillations which makes use of the generating feature of the audion, but does not employ the beat phenomena. The amplifying ratio of the audion depends more or less directly on the value of the wing current, and by varying this current periodically there will be a corresponding periodic change in the amplifying power of the audion. Hence an audion arranged to repeat a continuous wave under such conditions will produce in its wing circuit oscillations which vary periodically in amplitude, and which may therefore be received by a simple audion system. The first audion may be arranged to produce the necessáry variation in its amplifying power in the manner indicated in Figure 21, which also

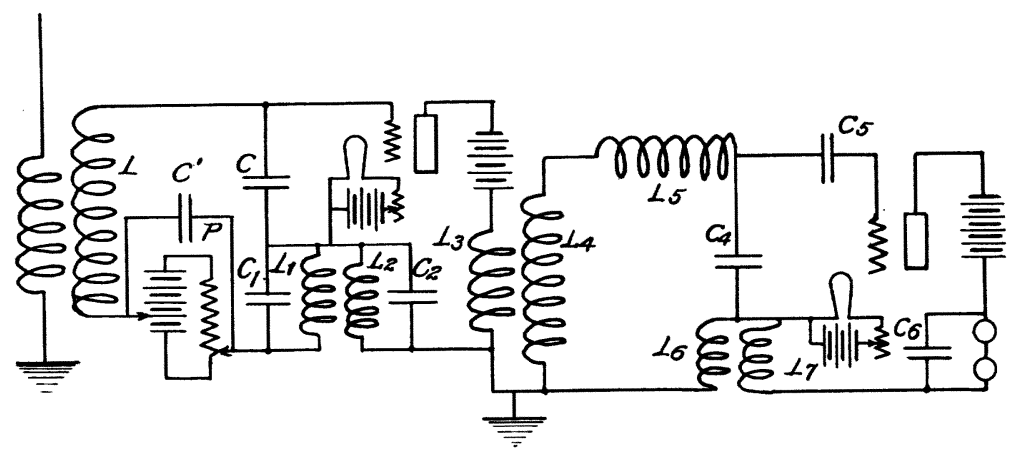

Figure 21

shows the complete circuits for carrying out this method of reception. Here $\mathrm{C}_{1} \mathrm{~L}_{1} \mathrm{~L}_{2} \mathrm{C}_{2}$ is an audio frequency system designed to produce audio frequency oscillations; and $\mathrm{P}$ is a potentiometer for adjusting the potential of the grid so that on the negative part of the oscillation in the wing circuit, the wing current is reduced practically to zero. The radio frequency 
circuit $\mathrm{C}^{\prime} \mathrm{L} \mathrm{C} \mathrm{C}_{1}$ is tuned to the oscillation frequency of the incoming wave. The radio frequency oscillations cannot be detected in the first audion system as the strong audio frequency current circulating in this system would produce a continuous note in the telephone receivers of such strength as to render inaudible all save very strong signals. By arranging to detect the oscillations in a second audion system coupled to the wing circuit of the first, interference of this sort is avoided; as the circuit $\mathrm{L}_{4} \mathrm{C}_{4}$ has a very high impedance for the audio frequency currents and the effect produced thru the magnetic coupling of $\mathrm{L}_{3}$ and $\mathrm{L}_{4}$ on the second system is negligible. The capacity current between these two coils thru the telephones to ground is, however, appreciable; and to avoid it it is advisable to ground their two adjacent ends as shown. The action of the system may be summed up as follows. The first audion system varies the amplitude of the incoming radio frequency oscillations at an audio frequency, and the second audion system amplifies and detects the radio frequency oscillations supplied to it by the first system. Diagrammatically, the phenomena occurring are as illustrated in Figure 22. The system gives about the same

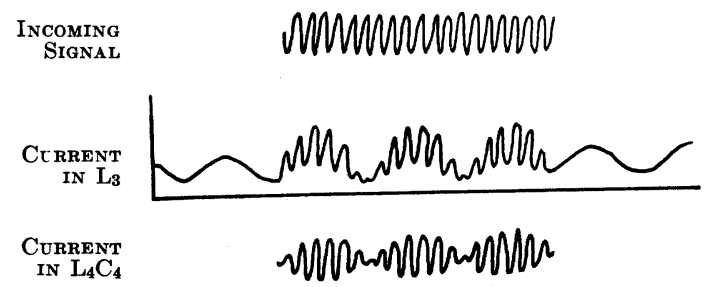

Figure 22

response as can be obtained with a single audion working with the beat method of reception. The advantages derived from the heterodyne method of amplification and the dependence of the audio frequency note in the receivers on the wave length are, of course, lacking; but for the reception of waves having a frequency higher than that at which beat reception is practicable, this method is of value.

\section{EFFECTS OF ATMOSPHERIC DISTURBANCES}

A very interesting feature of these receiving systems is their behavior under conditions of severe atmospheric disturbances, particularly when used for receiving continuous waves. Their success under such conditions is due to the fact that they com- 
bine in addition to their inherent property of responding more readily to a sustained wave than to a strongly damped one, the characteristics of the two most effective static eliminators known; the balanced valve and the heterodyne receiver. The function of the balanced valve is a physiological one, as it simply provides a means to shield the ear from the loud crashes which temporarily impair its sensitiveness for the relatively weak signals. In effect, it puts a limit on the noise which can be produced in the telephone by a stray, regardless of its amplitude. Now the effect of the static on an audion is to build up a negative charge on the grid, reducing the wing current, and the limit of the response which can be produced in the telephones is reached when the wing current is reduced to zero. Under ordinary conditions, this limit is too great to do much good; but when the audion is generating it is possible, by proper adjustment of the amplitude of the local oscillations, to reduce the wing current to a point just above the lower bend in the operating characteristic so that the audion is rendered insensitive to a further increase in the negative charge on the grid. The strays which cause serious interference are of a much greater amplitude than the local frequency, so that no appreciable interaction between the two takes place, and the wing current is invariably decreased. Since the decrease in the wing current is not in proportion to the change in the grid potential, the response in the telephone and the effect on the ear of the operator are correspondingly reduced. Static of smaller amplitude than the local oscillations may interact with them to produce either an increase or a decrease in amplitude of the oscillations in the grid circuit and may therefore cause either a decrease or an increase in the wing current. The wing current can, of course, increase to a relatively large value, but as it is impossible for the wing current to increase faster than the charge in the grid condenser can leak off, the rate of increase is necessarily slow. The response in the telephones is therefore not so disturbing as would be caused by a decrease of similar value where the rate of change of current is usually large.

When the system is operated without an auxiliary leak around the grid condenser, a peculiar paralysis of the audion is frequently caused by heavy static, no sound of any kind being heard in the telephones for a considerable length of time. If the apparatus is not touched, the paralysis may last for many minutes, and then suddenly disappear and the former sensitiveness be restored. The effect is primarily caused by the 
charging of the grid condenser to a sufficient potential to cut off entirely the flow of electrons to the wing, thereby decreasing the wing current to zero. Now the way in which the negative charge in the grid condenser leaks off is chiefly by means of the positive ions in the tube, which are drawn into contact with the grid when it becomes negatively charged. These positive ions are the result of ionization by impact, and when the voltage of the wing battery is properly adjusted, they can be produced only in the region between the grid and the wing, since the velocity attained by the electrons between the filament and grid is very low. When the grid is charged to a high negative potential it keeps all the electrons between the grid and filament, thereby barring them from the region between grid and wing. Hence the production of positive ions must cease and the usual means of removing the negative charge from the grid vanishes. The resistance of the leakage path of the grid condenser must then be almost infinite, as is shown by the very long time taken for the charge to leak from a condenser of approximately 0.0001 microfarads capacity. The effect is naturally the more pronounced the higher the vacuum, as the number of positive ions present is correspondingly reduced. A resistance of several hundred thousand ohms placed across the grid condenser gives a leak which is independent of the value of the wing current and which effectually prevents trouble of this kind. With the very high vacua now obtainable by the use of a molecular pump, there are practically no positive ions present so that the auxiliary leak is always necessary. Under these conditions, it not only prevents paralysis by the static but it also removes from the grid condenser the excess of negative electricity which accumulates in it, thereby increasing the sensitiveness of the audion and the sharpness of the signals in the telephones. The very high potentials to which the grid condenser may be charged by the static when it is not provided with an auxiliary leak are surprising. These potentials may be measured in a very simple and accurate way, here described. After a stray has cut off the wing current, if we continuously increase the capacity of the grid condenser the potential across it, and hence the potential of the grid, with respect to the filament, will be decreased inversely as the capacity. A point will finally be reached where the grid potential is sufficiently reduced to allow the wing current to flow. When this occurs it indicates that the potential of the grid condenser is slightly less than that shown by the operating characteristic as necessary to 
reduce the wing current to zero. The potential to which the grid condenser was originally charged is equal to th s voltage times the ratio of the capacity of the condenser at which the wing current began to flow to the original capacity. Voltages of over a hundred are not uncommonly reached by the grid; and as one volt represents a very strong signal, the difficulties of the static problem are very forcibly presented.

The fact that static of large amplitude produces almost invariably a decrease in the wing current while a signal (with beat reception) produces alternately an increase and decrease in the wing current is a circumstance of which it should be possible to take advantage. The circuits can be arranged to rectify the wing current in such a way that only the increases in this current are available to produce a response in the telephones, but in carrying this method out, trouble is experienced from a shifting zero. A better way of making use of the difference in response is the following one. Suppose that we arrange two complete receiving systems oscillating in step with each other, but so related to the antenna that the beat currents in the two systems are 180 degrees apart. The result of this will be that at the instant when the incoming signal is producing an increase of current thru the telephones in one receiver, it will be producing a decrease of current thru the telephones of the other receiver; so that the two telephone currents are 180 degrees out of phase. Static of large amplitude does not interact with the local frequencies, and will produce simultaneously in each receiver a decrease in the telephone current. These two currents are therefore in phase with each other. On replacing each telephone by the primary of a transformer, and connecting their secondaries thru a telephone in the proper phase, it is possible to balance out the static and at the same time secure an additive response of the signals from each receiver.

An arrangement of circuits by means of which this method can be carried out is shown in Figure 23. Here two oscillating receiving systems are kept in step by means of the circuits $\mathrm{L}_{1} \mathrm{C}_{1} \mathrm{C}_{1}{ }^{\prime} \mathrm{L}_{1}{ }^{\prime} . \quad \mathrm{L}_{1} \mathrm{C}_{1}$ and $\mathrm{L}_{1}{ }^{\prime} \mathrm{C}_{1}{ }^{\prime}$ are identical, and each is tuned separately to the frequency to be received. When both audions are oscillating in step, the flow of current in these circuits as indicated by the vectors of Figure 23 will be alternately up on one side and down on the other. The point between the condenser $\mathrm{C}_{1}$ and $\mathrm{C}_{1}{ }^{\prime}$ will be a node; and the antenna may be connected to this point without disturbing the conditions appreciably if a resistance $R$ placed as indicated is included in the 
antenna. This resistance need not be large enough to interfere seriously with the signal strength; it need only be large with respect to the resistance of the circuit $\mathrm{L}_{1} \mathrm{C}_{1} \mathrm{C}_{1}{ }^{\prime} \mathrm{L}_{1}{ }^{\prime}$, which circuit has a very low resistance. .

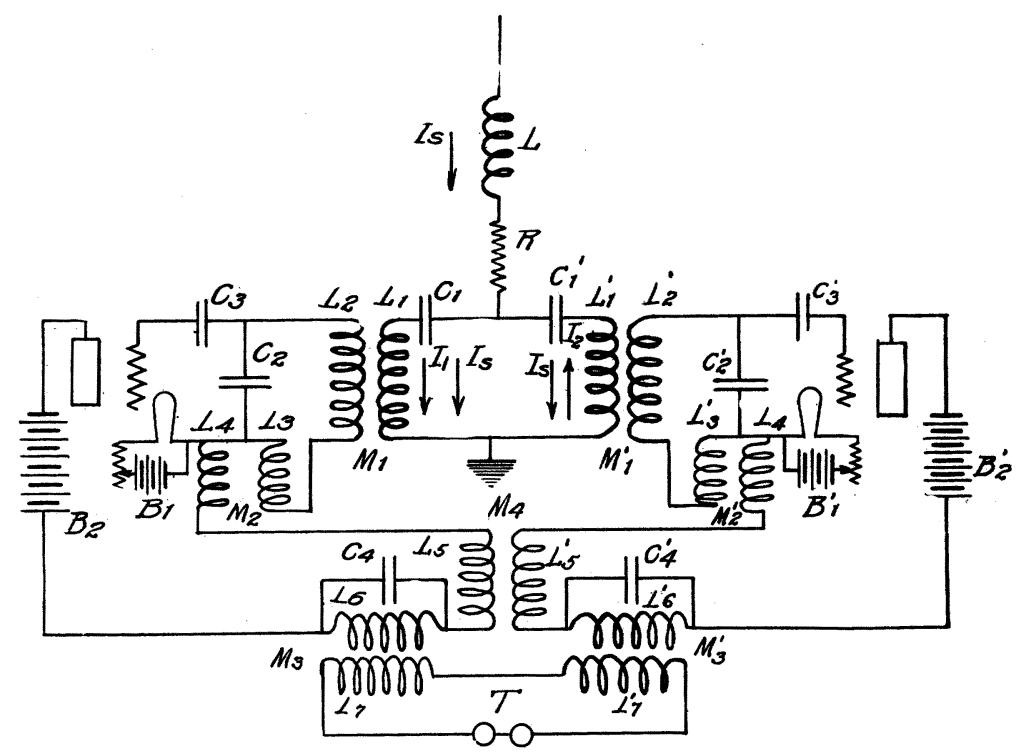

Figure 23

Incoming oscillations pass thru the divided circuit as indicated in the diagram, and therefore are in phase with the local oscillations of one receiver and 180 degrees out of phase with the local oscillations of the other. This produces the desired result in the currents thru the transformers of the circuit $\mathrm{T}$ which act in the manner already described.

It is found in practice that the oscillations set up in each system by the incoming signals tend to neutralize each other thru the circuit $\mathrm{L}_{1} \mathrm{C}_{1} \mathrm{C}_{1}{ }^{\prime} \mathrm{L}_{1}{ }^{\prime}$. This effect is avoided by introducing in the wing circuits a differential coupling arranged to neutralize the coupling between the two grid circuits. It is possible to do this, as it does not affect the coupling of either receiver with the antenna, and does not interfere with the local operation until the effective coupling between the two systems is reduced to a point below which they will no longer remain in step. There are other ways of securing the same result, but the system shown will illustrate the general procedure in carrying out this method of balancing. 
The practical results obtainable with these receivers may perhaps be of interest. At the present time, signals from all high power stations from Eilvese (Germany) to Honolulu are heard day and night at Columbia University with a single audion receiver. Cascade systems give correspondingly better results, two stages being sufficient to make the night signals of Honolulu audible thruout the operating room. Interference with the signals from Nauen by the arc station at Newcastle, New Brunswick (Canada), is very easily eliminated by means of an audio frequency tuning circuit; and this is the most severe interference we have yet experienced, the two frequencies sometimes differing by less than 1 per cent. and the arc signals being much the stronger.

These receivers have been developed in the Research Laboratory of Electro-Mechanics, Columbia University; and are mainly the result of a proper understanding and interpretation of the key to the action of the audion; the grid potential-wing current curve. In conclusion, I want to point out that none of the methods of producing amplification or oscillation depend on a critical gas action; they depend solely on the relay action of the tube employed (electron or gas relay) and the proper arrangement of its controlling circuits.

SUMMARY : The action of the audion as a detector and simple amplifier is explained, with the method of verification of the theory by means of oscillograms. To reinforce the oscillations in the grid circuit two methods are employed : first, to couple the grid circuit to the wing circuit and arrange the latter to permit radio frequency currents to pass freely in it; and second, to use a large inductance in the wing circuit, thereby tuning it to the incoming frequency (in conjunction with the capacity between the filament and wing in the audion itself). Both methods may be used together. Various methods of coupling grid and wing circuits are shown. Methods of combined audio and radio frequency amplification are described.

The audion, being a generator of alternating current of any desired frequency, can be used as a beat receiver. A steady audion generator of regular groups of radio frequency oscillations is illustrated. Various methods of audio frequency tuning permitting high selectivity are possible. By the use of two audions in cascade, amplifications as high as 1,000 are attainable. The cascade systems can be arranged so as to operate both audions either synchronously or non-synchronously.

As an alternative to beat reception of sustained wave signals, an arrangement is explained wherein the amplifying ratio of a repeating audion is varied periodically at an audio frequency. Coupled to this system is a simple audion detector. Musical signals of any desired pitch are thus obtained.

It is found that static of large amplitude nearly always decreases the wing current, while a signal (with beat reception) alternately increases and decreases it. A system of circuits is described whereby this fact is taken advantage of in balancing out static while retaining an additive response to signals, thus effecting an elimination of static to a considerable extent.

Finally, instances of long distance stations received and interference overcome in practice are given. 